\title{
Review Article: Breast Calcifications on Mammography
}

\section{ISSN: 2637-773X}

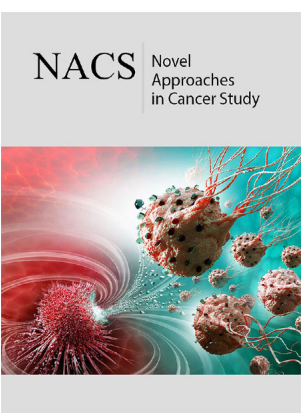

*Corresponding author: Kameswari P, Consultant Radiation Oncologist, Healthcare Global Enterprises Limited, Bangalore, India

Submission: 师 June 16, 2021

Published: 鲇July 26, 2021

Volume 6 - Issue 2

How to cite this article: Kameswari P*, Sudhakar S, Avinash K, Lohith G. Review Article: Breast Calcifications on Mammography. Nov Appro in Can Study. 6(2). NACS.000632. 2021.

DOI: 10.31031/NACS.2021.06.000632

Copyright@ Kameswari P, This article is distributed under the terms of the Creative Commons Attribution 4.0 International License, which permits unrestricted use and redistribution provided that the original author and source are credited.

\author{
Kameswari P*, Sudhakar S, Avinash K and Lohith G
}

Radiation Oncologist, India

\begin{abstract}
Breast calcifications are the most common finding on mammograms and can show significant morphological variability. With the help of the BIRADS (Breast Imaging Reporting and Data System) lexicon they can be divided into benign and malignant types but there remains overlap between these two categories. Micro calcifications which fall in the 'suspicious' category can occur without the presence of an associated mass or architectural distortion. Micro- calcifications are also one of the first features to be seen on mammograms in DCIS (Ductal Carcinoma In Situ), leading to early diagnosis and prompt treatment. With the advent of newer technologies in imaging, more information regarding the radiological extent of disease and cancer type associated with the calcification can be determined. This review aims to cover the BIRADS fifth edition descriptors and lexicons of breast calcifications on mammography.
\end{abstract}

\section{Background}

Calcifications are a common entity on mammograms, with most being benign. With the evolution in mammogram technology and screening programmes, microcalcifications have become easier to detect with reporting of DCIS increasing, thereby reducing patient mortality. With this review paper, we aim to review the imaging descriptors of benign and malignant calcifications seen on mammograms with a pictorial representation and brief literature review.

Keywords: Breast imaging; Mammography; BIRADS; Calcifications; Microcalcifications

Abbreviations: ACR-BIRADS: American College of Radiology -Breast Imaging and Reporting Data System; DCIS: Ductal Carcinoma In Situ; FFDM: Full Field Digital Mammography; DBT: Digital Breast Tomosynthesis; CC \& MLO: Cranio-caudal and Medio-lateral oblique; 3D: 3 Dimensional

\section{Introduction}

Calcifications are a common finding in the breast, and they can appear as unequivocally benign, unequivocally malignant, or indeterminate. Around $50 \%$ of invasive cancers are associated with microcalcifications and nearly a third of women recalled in screening programs, have only calcifications as a sign of cancer [1,2]. Hence identification and characterization of these calcifications is essential for assessing risk of malignancy and early detection of cancer. Mammography continues to be the most sensitive modality for the detection and differentiation of calcifications. The introduction of DBT (Digital Breast Tomosynthesis) along with FFDM (Full Field Digital Mammography), has further increased sensitivity and resolution. The use of BIRADS lexicon for assessing morphology, size and distribution of calcifications standardizes the reporting and enables better communication between radiologists and referring physicians. Our review covers the BIRADS descriptors for calcifications, their varied imaging appearances, and suitable recommendations.

\section{Breast Calcifications: Pathogenesis}

In the ducts and lobules of the breast, calcifications are formed by calcium deposition within mucin accumulating in the duct, lobular acini or cavities associated with the lesion. These calcifications can appear in low to intermediate grade DCIS or benign conditions such as sclerosing adenosis and ductal hyperplasia. They take a round or hazy/amorphous shape with size of the calcification depending on the size of the duct. Calcifications within the ducts and lobules can also be formed by the calcium deposition within cell debris and 
secretions within the lumen taking the shape of the duct as linear or sometimes granular microcalcifications, commonly seen in high grade lesions [3]. Calcifications can also occur in the stroma of fibrous lesions, such as in a fibroadenoma or in the stromal change of a malignant process [3]. And finally, calcifications can also result from calcium deposition within collagen as response to post-traumatic or post therapeutic steatonecrosis and within a hematoma. Calcium deposition in the breast can be categorized according to their chemical composition into Type I and Type II. In Type I, the calcific deposits are made of calcium oxalate and are mostly found in benign conditions. In Type II, the calcific deposits are made of calcium phosphate, and these are found in both benign and malignant conditions [4].

\section{Imaging Appearances of Calcifications}

Calcifications on mammography are characterized as either benign or suspicious and based on their distribution (Table 1). Benign calcifications are typically larger, coarser, round and have smooth margins. They are more commonly and easily seen than calcifications associated with malignancy. On the other hand, malignant calcifications are typically small and frequently need the use of magnification to be identified. When a typical benign aetiology cannot be assigned, description of calcifications should include their morphology and distribution.

Table 1: Imaging descriptors of calcifications.

\begin{tabular}{|c|c|c|}
\hline \multicolumn{2}{|c|}{ Calcifications } \\
\hline Typically Benign & $\begin{array}{c}\text { Suspicious } \\
\text { Morphology }\end{array}$ & Distribution \\
\hline 1.Skin & $\begin{array}{c}\text { 1.Amorphous } \\
\text { 2. Vascular }\end{array}$ & 1.Diffuse \\
\hline 3. Coarse, 'popcorn' & 3.Pleomorpheneous & 2. Regional \\
\hline 4.Large rod-like & $\begin{array}{c}\text { 4. Linear/Fine Linear } \\
\text { Branching }\end{array}$ & 3. Grouped \\
\hline 5. Round/Punctate & & 4.Segmear \\
\hline 6.Rim & & \\
\hline 7.Dystrophic & & \\
\hline 8.Milk of Calcium & & \\
\hline 9.Suture & & \\
\hline 10.Breast Infarcts & & \\
\hline
\end{tabular}

\section{Definition of micro calcifications}

Calcifications are categorized as microcalcifications when the diameter of the calcification is $<1 \mathrm{~mm}$ [3]. These are frequently associated with a malignant process. All macro-calcifications start as micro-calcifications and differentiation between benign and malignant types in the early stages can be difficult.

\section{Benign Calcifications}

\section{Skin}

Commonly seen along the infra-mammary fold, parasternal region or over the axilla they are round with central lucency, $<5 \mathrm{~mm}$ in size and appearing in tight groups. Additional mammographic views tangential to the skin may be required when they mimic suspicious calcifications near the skin surface [5]. Digital Breast Tomosynthesis, due to its quasi-3D nature can help make the identification of skin calcifications easier since the skin appears at the first and the last three images of the sequence.

'Tattoo Sign'- A useful sign in demonstrating the superficial location of skin calcifications. In this sign, the position of the calcifications does not change on multiple views [6] (Figure 1a \& 1b).

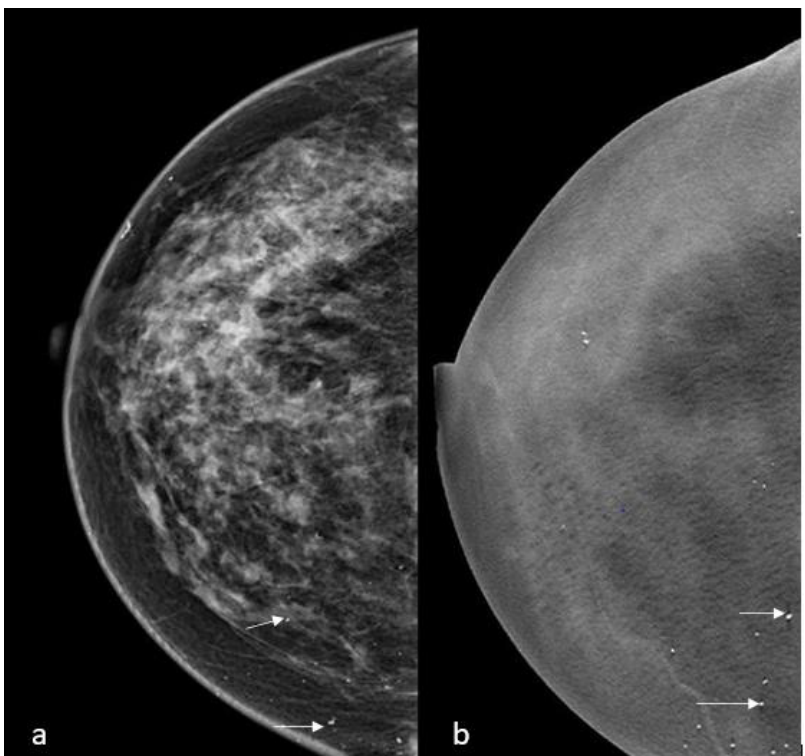

Figure 1: a) Skin calcifications(arrows) noted in the CC FFDM and DBT view of the right breast of a 50-year-old woman. b) Digital Breast Tomosynthesis images aid in the diagnosis of skin calcifications as they are seen in the first or last three of the images in the stack. Image Courtesy: HCG Hospitals, Bangalore.

\section{Vascular}

These unequivocally benign calcifications are frequently seen in the older age group. These appear as parallel tracks ('Tram track') or as linear tubular calcifications. The serpentine vascular course helps to differentiate it from the ductal branching pattern. Discontinuous calcifications, forming on only one side of the vessel wall might give the appearance of intra- ductal calcification or may not show a clear association with a vascular structure. In these cases, additional magnification views may be needed $[5,7,8]$ (Figure 2). 


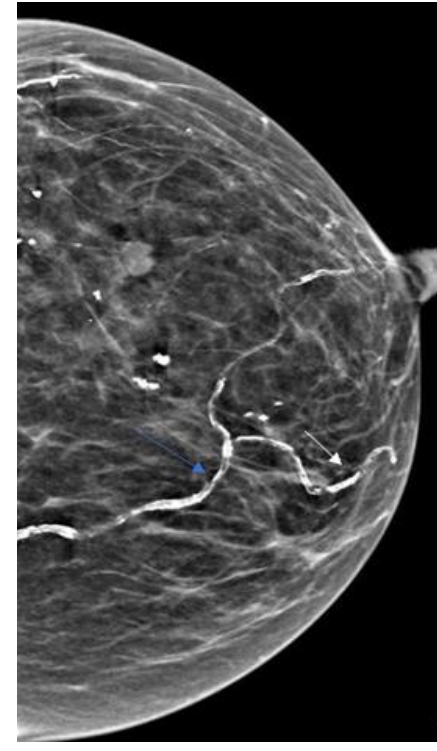

Figure 2: The above image is a LMLO view showing extensive vascular calcifications (arrows) in a 94-yearold woman. Image Courtesy: HCG Hospitals, Bangalore.

\section{Coarse/popcorn like}

These calcifications are most often seen within involuting fibroadenomas. The calcifications in fibroadenomas generally begin in the periphery as small calcifications measuring $>2-3 \mathrm{~mm}$ and coalesce over time to produce the coarse classic 'popcorn' calcifications [5].

Important differential: Mucinous carcinoma can also rarely show popcorn type calcification [9]; (Figure 3).

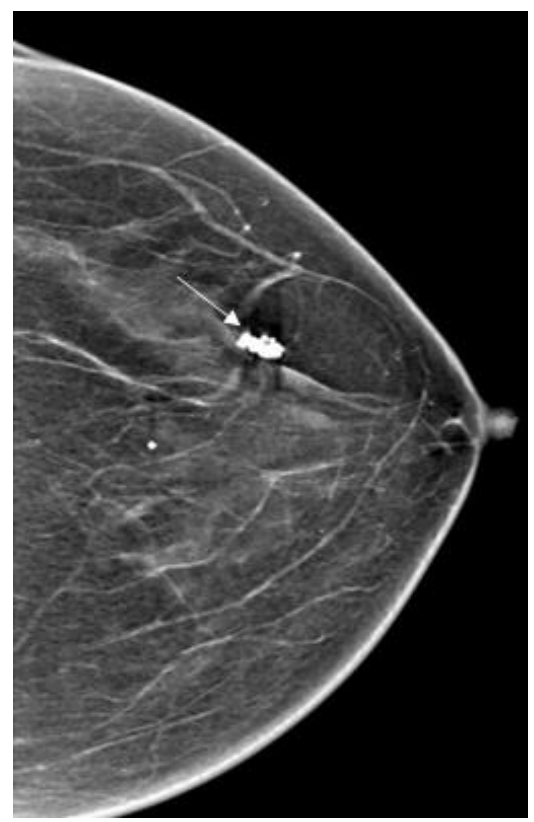

Figure 3: LCC image taken of a 68 year old woman showing coarse, 'popcorn' calcification (long arrow) in an involuting fibroadenoma. (Image courtesy; HCG Hospitals, Bangalore).

\section{Large rod-like or secretory}

These benign calcifications are seen in about $3 \%$ of mammograms and appear as smooth, large, linear discontinuous rod like calcifications measuring more than $0.5 \mathrm{~mm}$. They can be intra- ductal, appearing solid or periductal showing a central lucency with a characteristic ductal or ductal branching distribution radiating towards the nipple. These are commonly seen in the elderly and involving both the breasts. They can sometimes cause a diagnostic dilemma when seen unilaterally as few calcific particles [5-7]. Differentiation from malignancy is typically straightforward since in DCIS, the calcifications although linear are more irregular, do not have lucent centres, are thinner and more discontinuous [8]; (Figure 4).

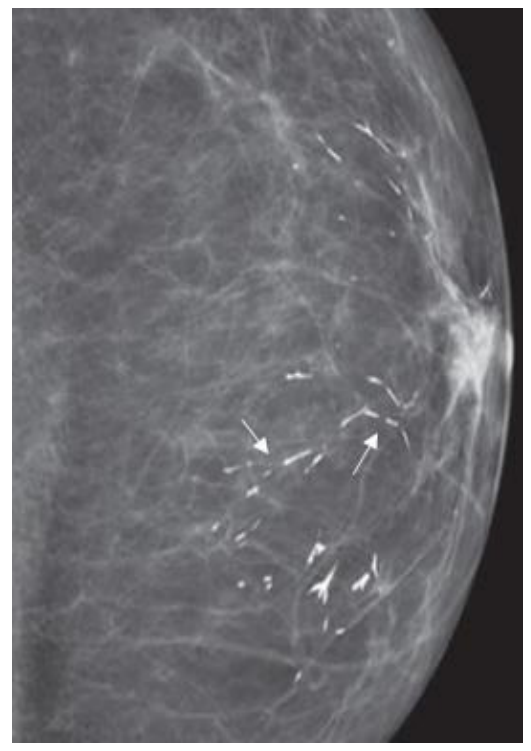

Figure 4: LCC view showing smooth rod like linear calcifications in a 76-year-old woman (long arrows). Image courtesy [8].

Round and punctate calcifications: These calcifications, having varying sizes and originate within the acini of lobules as calcium oxalate deposits. They are called round when greater than $0.5 \mathrm{~mm}$ and punctate (subset of round) when less than $0.5 \mathrm{~mm}$. When they are isolated or diffuse with no prior mammogram, they can be considered benign. They can be assigned the BIRADS 3 category if they are mostly grouped with short term monitoring at 6 months $[5,6]$. Biopsy may be warranted if the grouped calcifications are new, increasing, linear or segmental in distribution or when found adjacent to a cancer focus.

It is helpful to remember a few differential diagnoses for round and punctate calcifications as they appear in a variety of cases. When these calcifications have a lucent centre, they are most likely skin calcifications, oil cysts or evolving dystrophic calcifications. Oil cysts show the milk of calcium appearance where it appears 'smudgy' and hazy on CC (Cranio-Caudal) views and showing the 
typical layering of the calcium on the lateral views. Skin talc can also mimic round calcifications and they disappear once the area is cleaned. Punctate calcifications can sometimes be seen in DCIS, although with some heterogeneity and should be carefully assessed [7] (Figure 5).

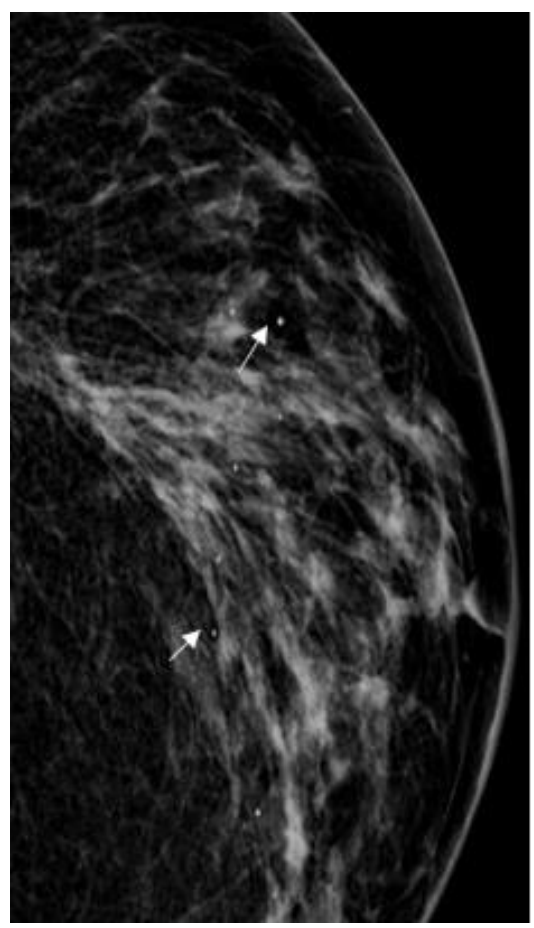

Figure 5: The above LMLO view of a 56-year-old woman shows diffuse punctate/ round calcifications (long arrows). Image courtesy: HCG Hospitals, Bangalore.

\section{Rim calcifications}

Previously called 'Eggshell/Lucent centred', they are benign, non-grouped, thin and appear as 'calcium deposited on the edge of a sphere'. Initially these lesions are seen as round or oval lucent lesions that eventually develop a calcified spherical surface with a lucent centre. The calcific deposits when viewed on edge measure less than $1 \mathrm{~mm}$ in thickness but can range in size from less than $1 \mathrm{~mm}$ to more than a centimetre. They occur more commonly in a superficial location along with a history of trauma or surgery but are also known to occur spontaneously in large breasts $[5,6]$.

On DBT, these thin calcifications which are curvilinear are better depicted on the walls of oil cysts along with associated fat density [10]; (Figure 6).

\section{Dystrophic calcifications}

These calcifications are large, thick, and irregular in shape with many found to have irregular central lucency indicating the presence of fat. They are secondary to fat necrosis occurring in response to trauma, surgery, or irradiation to the breast. When occurring after surgery they can be usually found near the surgical scar forming around 3- 5 years after the operation [5,6]; (Figure 7).

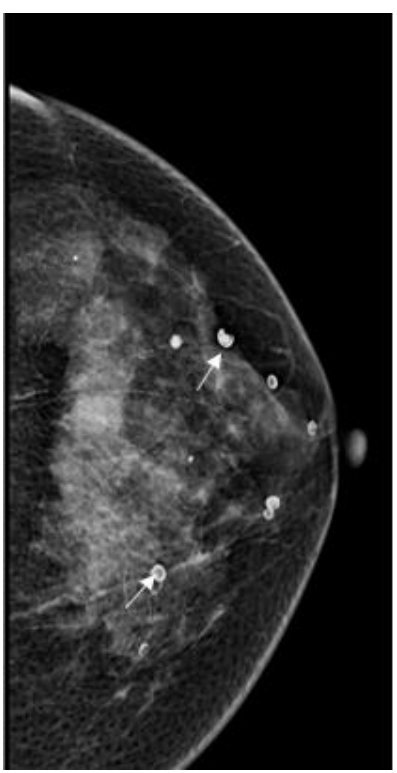

Figure 6: LMLO view of a 67-year-old woman showing multiple rim and curvilinear calcifications of circumscribed lesions with lucent centres. Image courtesy: HCG hospitals, Bangalore.

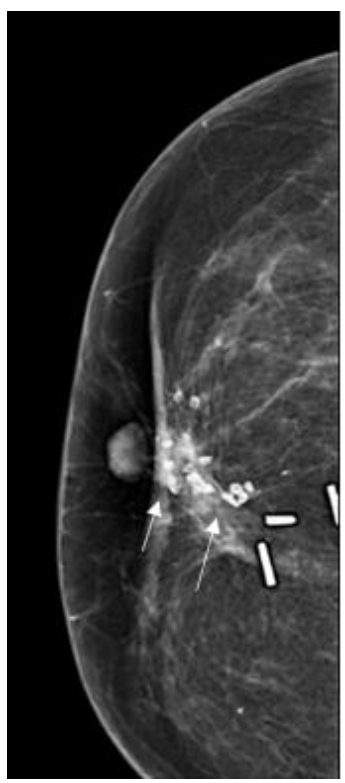

Figure 7: $\mathrm{CC}$ view demonstrating multiple dystrophic calcifications in a regional distribution in a post-operative right breast. Image courtesy: HCG Hospitals, Bangalore.

\section{Milk of calcium}

Milk of calcium refers to the calcium deposits (usually calcium oxalate) formed in benign cysts (micro or macro) in fibrocystic disease. They commonly occur bilaterally and in the menopausal/ peri-menopausal age group. On CC images, they appear round and 'smudgy', but when lateral 90-degree views are taken they appear crescentic, semi-lunar or curvilinear in shape ('tea-cup' configuration) with concave side pointing up and are occasionally 
seen on MLO (Medio-Lateral Oblique) views also, confirming their 'dependent nature'. This change in shape on different projections aids in their diagnosis [5,6]; (Figure 8a \& 8b).

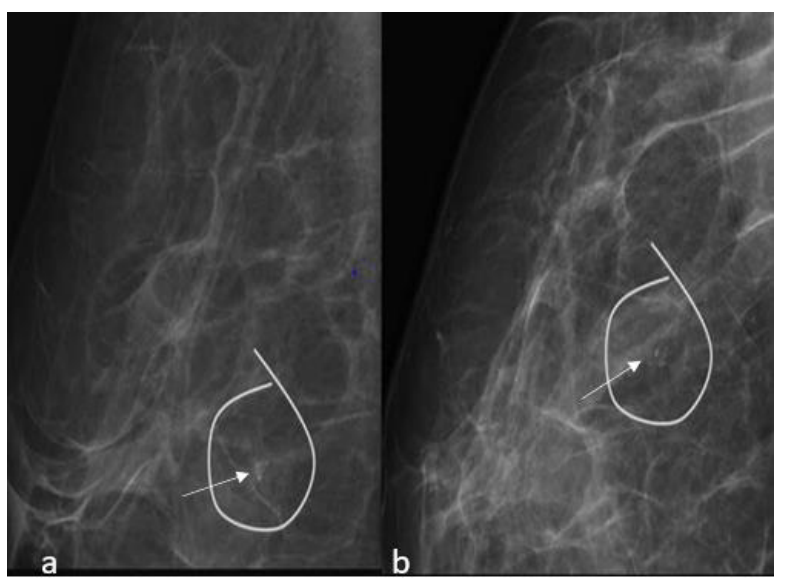

Figure 8: a) The above mammographic images show 'milk of calcium' calcifications on the lateral view(left) which appear smudged on the $\mathrm{CC}$ view(right). b) The calcifications are seen to be in the 'tea-cup' formation on the lateral view.

\section{Sutural}

Commonly occurring at surgical and biopsy sites of an irradiated breast as seen in many studies [11]. (Although they can also rarely occur in a non-irradiated post-surgical breast), they are categorized as benign due to their typical appearance (knotted configuration). In their early development they can be confusing with their vague and non-characteristic morphology and might be mistaken for possible recurrence. Additional magnification views showing the presence of calcifications along a sutural plane will help in diagnosis [8,12]; (Figure 9).

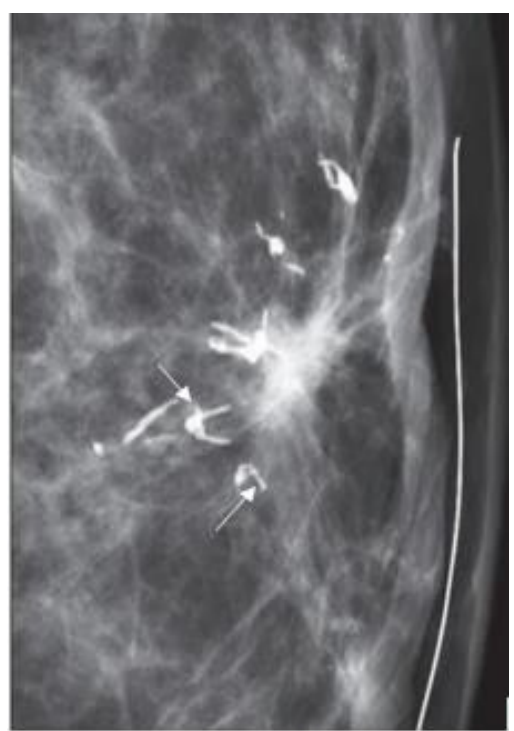

Figure 9: Magnified CC image showing the classic 'knotted configuration' seen in sutural calcifications (long arrows). Image courtesy [8].

\section{Breast infarcts}

Breast infarcts occur due to a variety of reasons and settings. For example, due to anti-coagulant therapy, in coronary artery bypass grafting using internal mammary artery, in pregnancy, in breast lesions like phyllodes tumour and fibroadenoma etc. In mammograms, they may appear as microcalcifications with a grouped distribution arousing suspicion for malignancy and need to be thoroughly evaluated [13]; (Figure 10).

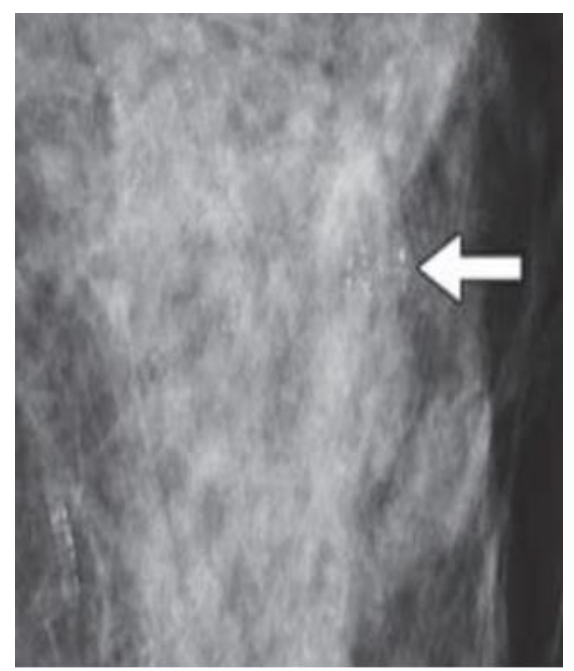

Figure 10: Magnified CC view taken of a 89 year old woman's breast showing grouped heterogeneous calcifications. The patient was on anti -coagulant therapy and due to the morphology of the calcifications, biopsy was done which revealed a breast infarct. Image Courtesy [12].

\section{Artifacts}

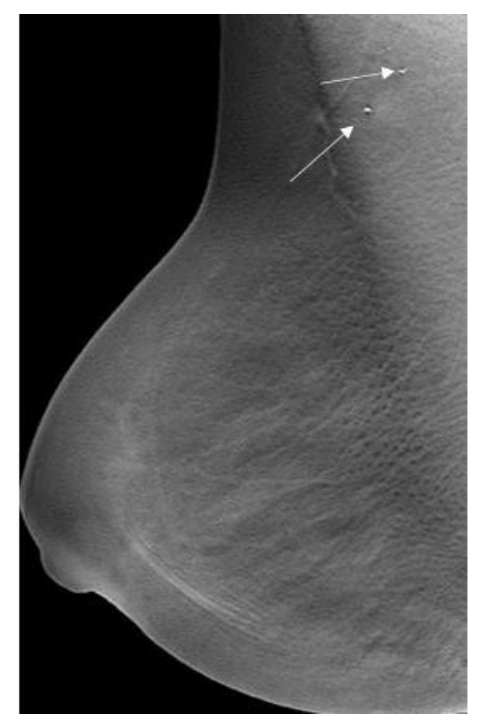

Figure 11: RMLO image of a 39-year-old woman showing radio-dense opacities in the axillary region, most likely caused by radio-opaque containing material like antiperspirant (long arrows). Image courtesy: HCG Hospitals, Bangalore. 
Many compounds which contain radio-opaque material like zinc, aluminium, magnesium can mimic calcifications on mammography causing concern to the patient and the radiologist. They can also mask the true underlying abnormalities. Some examples of substances which form pseudo-calcifications are various ointments, deodorants, and talc. Patients should be informed not to wear deodorants, ointments etc. before the mammogram [14]; (Figure 11).

\section{Malignant Calcifications}

\section{Amorphous}

They are small and hazy in appearance without a specific shape. When they occur in a grouped, linear, or segmental distribution, they are deemed suspicious and commonly warrant biopsy. But when they appear in both breasts and are diffuse, they can be assigned a benign BIRADS category. According to ACR BIRADS, amorphous calcifications have a positive predictive value of $20 \%$ and are therefore assigned the BIRADS $4 \mathrm{~b}$ category [5]. Many studies have been done on assessing the efficiency of mammography imaging techniques in the characterization of amorphous calcifications and the utility of biopsy of these calcifications.

In a study by Ferreira et al. [15] 78 patients with suspicious grouped amorphous calcifications on mammography and categorized as BIRADS 4 were retrospectively analysed with their histopathology results with samples having been obtained via VABB. Their conclusion was that amorphous calcifications of a suspicious nature showed a correlation more with precursor lesions, than with malignancy with a ratio of 3:1.

In another study by Oligane et al. [16] published in 2018, they retrospectively analysed 497 lesions from 494 patients that underwent stereotactic biopsies based on the presence of amorphous calcifications. In their study, they found that $10.5 \%$ lesions were malignant with an association of the invasive cancers and hormone receptor positivity (Figure 12).

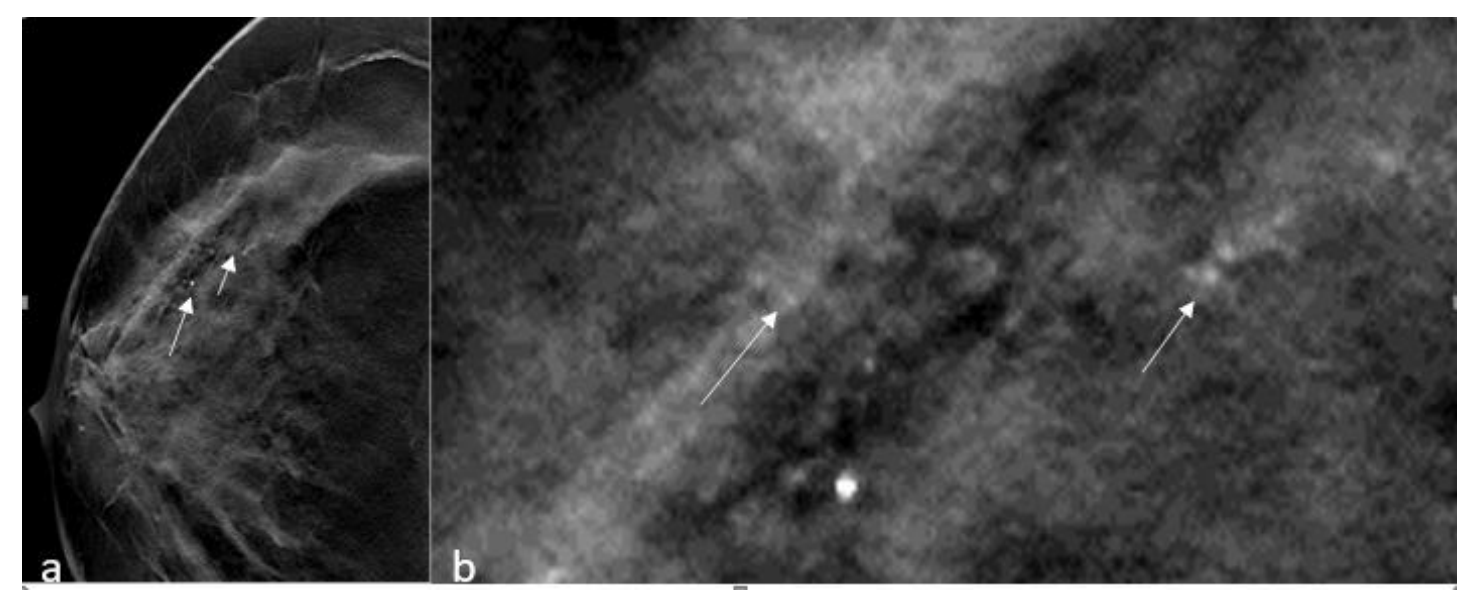

Figure 12: RMLO view and RMLO magnified view taken of a 64-year-old woman shows grouped amorphous calcifications. Image courtesy: HCG Hospitals, Bangalore.

\section{Fine pleomorphic}

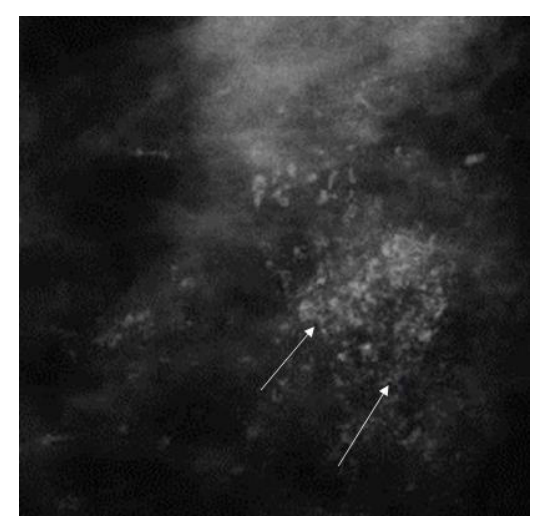

Figure 13: RMLO magnified view of a 42-yearold woman showing extensive pleomorphic microcalcifications(arrows). The diagnosis for this patient is DCIS with IDC. Image courtesy: HCG Hospitals, Bangalore.
These are more conspicuous than the hazy amorphous calcifications and tend to have discrete shapes and measuring $<0.5 \mathrm{~mm}$ in diameter. These calcifications are irregular and vary in size and shape. They have a higher positive predictive value of $29 \%$ for malignancy and are therefore categorized as BIRADS $4 \mathrm{~b}$ [5]; (Figure 13).

\section{Coarse heterogeneous}

These irregular calcifications are generally larger than pleomorphic calcifications measuring between $0.5 \mathrm{~mm}-1 \mathrm{~mm}$; commonly seen in fibroadenomas, fibrosis or areas of trauma eventually becoming dystrophic calcifications. When bilateral, groups of these calcifications, can be categorized as benign. When a single group of these calcifications is seen, they can be associated with malignancy and have a Positive predictive value of $<15 \%$, and hence are categorized as BIRADS 4b [5]; (Figure 14). 


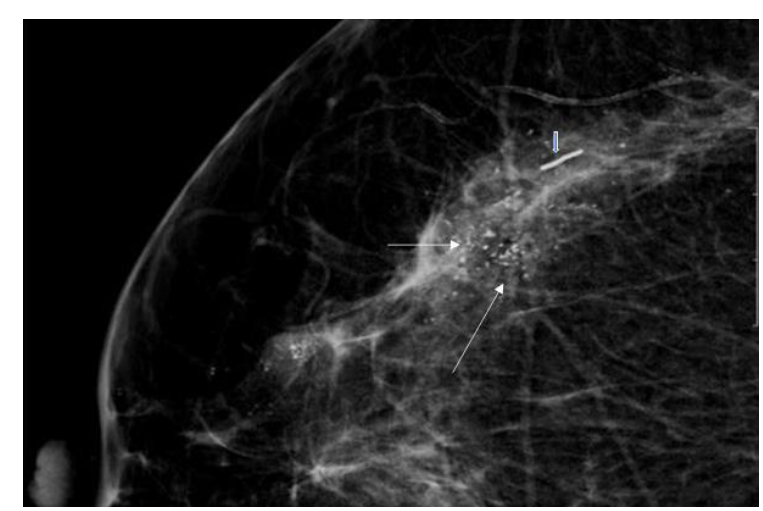

Figure 14: RCC zoomed in view of a 51-year-old woman with coarse heterogeneous calcifications (long arrows). A metallic marker is also seen (short arrow). Image Courtesy: HCG hospitals, Bangalore.

\section{Linear and fine linear branching}

As the name suggests they are fine, linear, thin, and irregular measuring $<0.5 \mathrm{~mm}$. They appear sometimes as discontinuous calcifications, as well as in a branching pattern, developing due to the filling in of the lumen of a duct/ducts which are involved by carcinoma. They have the highest positive predictive value amongst the suspicious calcifications (70\%). They are categorised as BIRADS 4C [5]; (Figure 15).

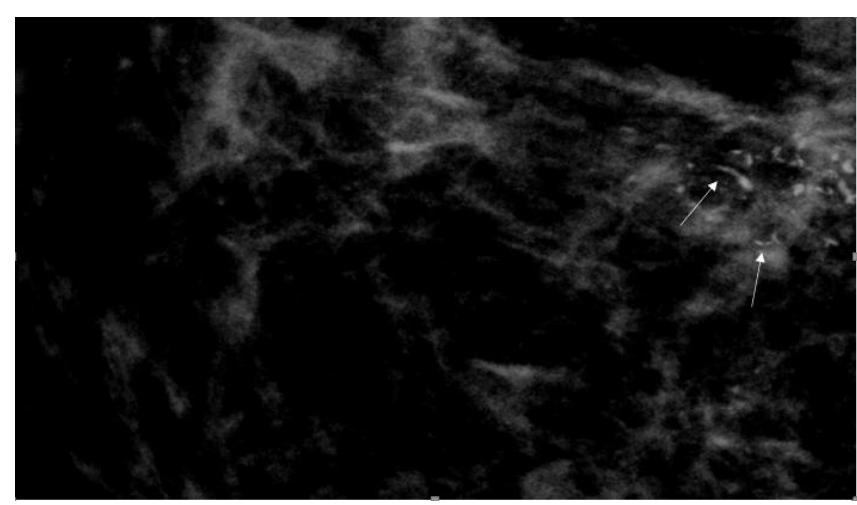

Figure 15: RCC view of a 61-year-old woman showing linear calcifications (long arrows). Image Courtesy: HCG Hospitals, Bangalore.

\section{Distribution of Calcifications}

The distribution of calcifications is as equally important as the morphology of calcifications.

A. Diffuse - Where calcifications are distributed randomly throughout the breast. They are almost always benign.

B. Regional - where $>2 \mathrm{~cm}$ (lower limit) of breast tissue is occupied. Malignancy is less likely since they do not have a ductal distribution.

C. Grouped (previously known as clustered) - Calcifications which are present in a small portion of breast tissue - lower limit is 5 calcifications within $1 \mathrm{~cm}$ and upper limit is a larger number of calcifications within $2 \mathrm{~cm}$.

D. Linear- arranged linearly along a duct. They can increase suspicion for malignancy when thin and irregular.

E. Segmental- deposits in a duct and their branches- they are twice as likely to be associated with malignancy [5,17-19].

\section{Summary}

Calcifications as seen above are an important indicator of breast disease, but trouble arises when delineating between benign and malignant forms. All forms of calcifications begin as microcalcifications and with advances in mammography, identification of micro-calcifications which have a suspicious morphology and distribution with subsequent HPE (Histo-Pathology Evaluation) confirmation of malignancy have significantly reduced the morbidity and mortality associated with breast cancer. The imaging and analysis of breast calcifications have become a subject of interest in the fields of artificial intelligence and spectroscopy as well especially when calcifications may present as the only indicator of malignant pathology. With the aid of BIRADS descriptors which are a standard format used the world over, this review hopes to be a revision of the terminology used in the fifth Edition of ACR BIRADS for not just radiologists, but also pathologists and surgeons dealing with breast disease.

\section{References}

1. Mordang JJ, Gubern-Mérida A, Bria A, Tortorella F, Mann RM, et al. (2018) The importance of early detection of calcifications associated with breast cancer in screening. Breast Cancer Res Treat 167(2): 451-458.

2. Paredes ES (2007) Atlas of mammography. Lippincott Williams \& Wilkins, ISBN:0781764335, Philadelphia, Pennsylvania, USA.

3. Henrot P, Leroux A, Barlier C, Génin P (2014) Breast microcalcifications: The lesions in anatomical pathology. Diagn Interv Imaging 95(2): 141152.

4. O'Grady S, Morgan MP (2018) Microcalcifications in breast cancer: From pathophysiology to diagnosis and prognosis. Biochim Biophys Acta Rev Cancer 1869(2): 310-320.

5. American College of Radiology Committee on BI-RADS ${ }^{\oplus}$ (Breast). ( $5^{\text {th }}$ edn), ACR BI-RADS ATLAS Breast Imaging Reporting and Data system.

6. Arancibia Hernández PL, Taub Estrada T, López Pizarro A, Díaz Cisternas ML, Sáez Tapia C (2016) Breast calcifications: Description and classification according to BI-RADS $5^{\text {th }}$ edition. Rev Chil Radiol 22(2): 80-91.

7. Itani M, Griffin AT, Whitman GJ (2013) Mammography of breast calcifications. Imaging Med 5(1): 63-74.

8. Lai KC, Slanetz PJ, Eisenberg RL (2012) Linear breast calcifications. Am J Roentgenol 199(2).

9. Berg WA, Leung J (2019) Diagnostic Imaging: Breast. ( $3^{\text {rd }}$ edn).

10. Horvat JV, Keating DM, Rodrigues-Duarte H, Morris EA, Mango VL (2019) Calcifications at digital breast tomosynthesis: Imaging features and biopsy techniques. Radio Graphics 39(2): 307-318. 
11. Stacey-Clear A, McCarthy KA, Hall DA, Pile-Spellman ER, Mrose HE, et al. (1992) Calcified suture material in the breast after radiation therapy. Radiology 183(1): 207-208.

12. Krishnamurthy R, Whitman GJ, Stelling CB, Kushwaha AC (1999) Mammographic findings after breast conservation. Therapy Radio Graphics (Suppl_1).

13. Demetri-Lewis A, Slanetz PJ, Eisenberg RL (2012) Breast calcifications: The focal group. Am J Roentgenol 198(4): W325-343.

14. Almeida Costa NA, Rio G, Garrido Santos J (2018) Suspicious breast pseudocalcifications. BMJ Case Rep.

15. Ferreira VCC de S, Etchebehere ECS de C, Bevilacqua JLB, Barros N de (2018) Suspicious amorphous microcalcifications detected on full-field digital mammography: Correlation with histopathology. Radiol Bras 51(2): 87-94.
16. Oligane HC, Berg WA, Bandos AI, Chen SS, Sohrabi S, et al. (2018) Grouped amorphous calcifications at mammography: Frequently atypical but rarely associated with aggressive malignancy. Radiology 288(3): 671-679.

17. https://radiologyassistant.nl/breast/bi-rads/bi-rads-formammography-and- ultrasound-2013.

18. de Lara Bendahan V, Ramos Solis MF, Amador Gil A, Gómez de las Heras C, Pérez Herrera JC, et al. (2014) Breast calcification: Management and pictorial review. Poster number: C-0692, Poster presented at: ECR 2014.

19. Chen PH, Ghosh ET, Slanetz PJ, Eisenberg RL (2012) Segmental breast calcifications. Am J Roentgenol 199(5). 